\title{
Er(III)-chelated Prototype Complexes Based on Benzoate and Pentafluorobenzoate Ligands : Synthesis and Key Parameters for Near IR Emission Enhancement
}

\author{
Soo-Gyun Roh, Jae Buem Oh, Min-Kook Nah, Nam Seob Baek, Youngil Lee, and Hwan Kyu Kim \\ Center for Sinart Light-Harvesting Materials and Department of Polymer Science \& Engineering. \\ Hannam Unwersit, 133 Ojung-dong, Daeduck-gu. Daejeon 306-791. Korea \\ ${ }^{\dagger}$ Dongbu Research Council, Daejeon 103-2, Korea \\ ReceivedMarch 24, 2004
}

\begin{abstract}
New synthetic methodology of the saturated and unsaturated Er(III)-chelated prototype complexes based on benzoate and pentafluorobenzoate ligands was developed through ligand-exchange reaction. The saturated 8coordinated Er(III) complexes exhibit stronger near- $\mathbb{R}$ emission than those of the unsaturated 6-coordinated Er(III) complexes. obtained from the direct photoexcitation of Er ions with $488 \mathrm{~nm}$. Three $\mathrm{H}_{2} \mathrm{O}$ molecules coordinated in the unsaturated 6-coordinated complexes seriously quenched the near $\mathbb{R}$ emisssion by the harmonic vibration relaxation decay of $\mathrm{O}-\mathrm{H}$ bonds. Also, the stronger emission of the $\mathrm{Er}(\mathrm{III})$ complexes was obtained by the indirect photoexcitation of ligands than by the direct photoexcitation of the $\operatorname{Er}(\mathrm{III})$ ions. due to the energy transfer between the excited ligand and the erbium ion. Furthermore, the saturated $\operatorname{Er}(\mathrm{III})$-chelated complex with C-F bonds shows much stronger near $\mathbb{R}$ emission than that of the saturated $\mathrm{Er}(\mathrm{III})$-chelated complex with $\mathrm{C}-\mathrm{H}$ bonds. It is attributed to the influence of $\mathrm{C}-\mathrm{F}$ bonds on near $\mathrm{R}$ emission.
\end{abstract}

Key Words : Erbium-chelated prototype complexes. Ligand-exchange reaction, Emission enhancement. Planar waveguide amplifiers

\section{Introduction}

Very recently. the lanthanide(III) materials play an important role in the development of integrated planar waveguide optical amplifiers in the superhigh speed communication systems. ${ }^{1}$ In particular, wavelength division multiplexing (WDM)-integrated planar waveguide amplifiers are recognized to be essential for successfully realizing photonic devices in WDM technology: However. the problem still remains to be unsolved in the materials for the planar waveguide antlifiers. At present. erbium-doped silica amplifiers are widely used. The optical amplification (OA) gain of erbium-doped waveguide amplifiers (EDWA) utilizing this material is currently not enough for amplifying the optical signals in small-sized photonic devices, although the $O A$ gain of current erbium-doped fiber amplifiers (EDFA) based on $\operatorname{Er}(\mathrm{III})$-doped silica materials is not a problem for intercontinental teleconmunication networks with long pathway. Therefore. in order to enhance the amplified luminescence intensity: the development of luminescent lanthanide complexes based on the energy transfer mechanisms by using the supramolecular ligands has been extensively studied ${ }^{->-7}$ Luminescent lanthanide complexes consist of a lanthanide ion as a photonic active component and its chelating luminescent ligands as sensitizers. which can transfer excitation energy from the ligands to the lanthanide ion. However. luninescent lanthanide complexes were not developed in specific reference to advanced photonic materials by now. They are simply

"To whom all correspondence should be addressed. Tel: $-82-42-629$ 7865: Fax: -82-42-629-8325: e-mail: hwankkima mail.hannam.ac.kr supramolecular complexes containing well-known antenna cluromophores to photoexcite the lanthanide ions via the energy transfer process. They were not satisfied with the quantum yield of energy transfer and the luminescence efficiency yet. Such efforts are just in the early stage. Not only the useful synthetic methodology as well as the basic concept has not been established. but also the structureproperty relationship is not clearly understood yet.'

Recently, we studied the synthesis and photophysical properties of the luminescent lanthanide complexes based on coumarin. ${ }^{\S}$ porphyrin ${ }^{9}$ and naphthalene ${ }^{|c|}$ derivatives for advanced photonic application, such as planar waveguide amplifiers and light-emitting diodes, in order to get the highly efficient lanthanide emission via the energy transfer between luminescent ligand and lanthanide ion.

Very recently. we also attempted to synthesize Er(III)chelated benzoate and pentafluorobenzoate complexes as prototype complexes in order to investigate important key parameters for near infrared (IR) emission enhancement (see Scheme 1). Er(III)-chelated prototype complexes were carried out by using the $\operatorname{Er}($ III) acetate. according to the previously reported synthetic method for the 6-coordinated erbium complexes. ${ }^{11}$ But, the successful synthesis of 6- or 8coordinated erbium complexes was not always achieved. ${ }^{12.13}$ Thus. we have attempted to develop a new synthetic method for 6- and 8-coordinated $\operatorname{Er}(\mathrm{III})$-cored prototype complexes through the ligand-exchange reaction using $\mathrm{ErCl}_{3}$. In this paper, therefore, we report the synthesis of novel $\operatorname{Er}(\mathrm{III})$ chelated complexes through the ligand-exchange reaction and the investigation of important key parameters for near infrared (IR) emission enhancement. 

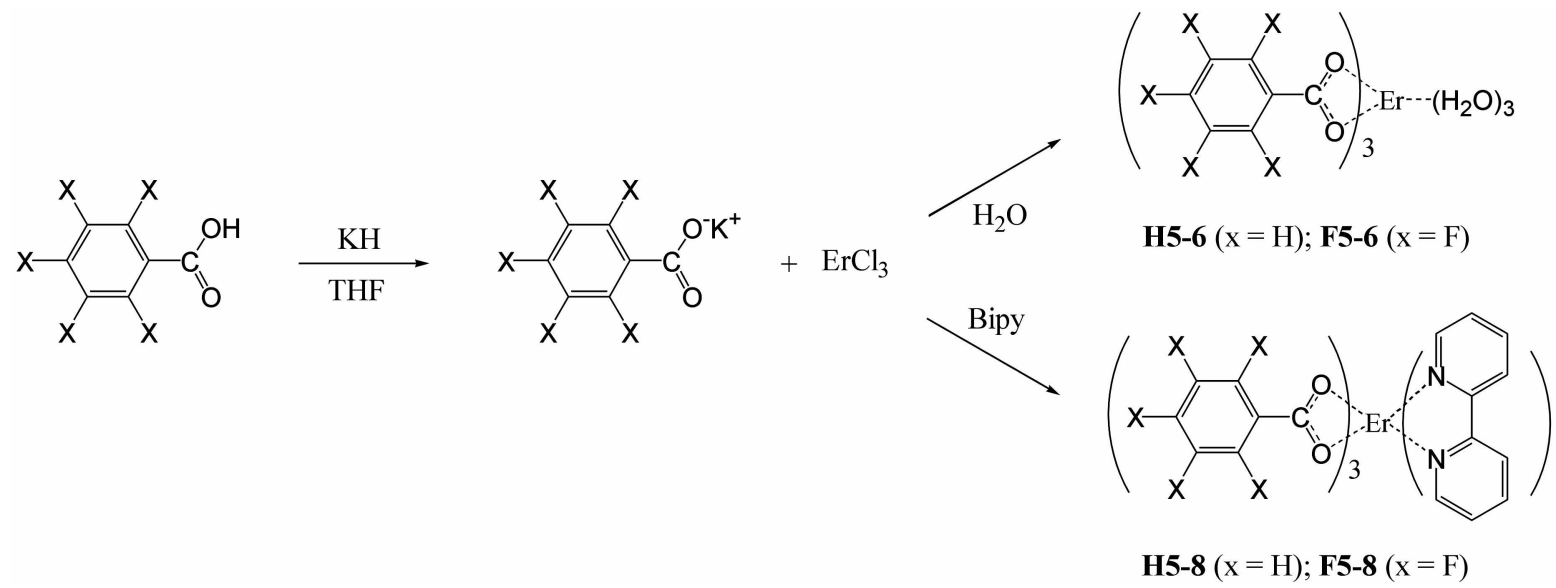

Scheme 1. New synthetic route to Er-chelated complexes based on benzoate and pentafluorobenzoate ligands and their chemical structures.

\section{Experimental Section}

General Procedures. All manipulations were carried out under $\mathrm{N}_{2}$ atmosphere with standard Schlenk technicue. All reagents were used as received without further purification. THF were dried and deoxygenated over sodium/benzophenone kethyl and freshly distilled prior to use. FT-JR spectrat were recorded on Perkin-Finner 1000 FT-IR spectrometer with a conventional $\mathrm{KBr}$ pellet method. UVvisible spectra were measured with Shimazu UV-2401PC spectrophotometer. TGA analysis was carried out with Dupont 9900 Model 951 thermogravimetric analyzer with the heat rate of $10^{\circ} \mathrm{C} / \mathrm{min}$ under $\mathrm{N}_{2}$ atmosphere.

Synthesis of $\mid \operatorname{Er}$ (benzoate $)_{3}\left(\mathrm{H}_{2} \mathrm{O}\right)_{3} \mid(5 \mathrm{H}-6)$. To the dried Schlenk flask, a mixture of benzoic acid $(0.500 \mathrm{~g}, 4.094$ mmol) and $\mathrm{KH}(0.164 \mathrm{~g}, 4.094 \mathrm{mmol})$ in THF (100 $\mathrm{ml}$ ) was added and stirred at room temperature for overnight. The salt was precipitated with gas evolution during reaction and then the methanol solution of anhydrous $\mathrm{FrCl}_{3}(0.373 \mathrm{~g}$. 1.364 mmol) in methanol $(10 \mathrm{~mL})$ was added dropwise to the reaction solution. After 2 days of stirring at room temperature, the resulting solution was filtered and removed solvents to give the residue. This residue was redissolved with $\mathrm{IHF}$ and the insoluble solid was filtered off. The solvent was removed in vacuo to give a solid. The solid was washed with n-hexane and diethyl ether, and then dried to afford 5H-6 as a white solid (0.502 g. $0.859 \mathrm{mmol}, 63 \%)$. Anal. $(\%)$ Calc. for $\mathrm{C}_{21} \mathrm{H}_{21} \mathrm{ErO} \%$ : $\mathrm{C} 43.14, \mathrm{H}$ 3.62. Er 28.61; Found: C, 43.01, H 3.76, tr, 28.55. UV-vis. ( $\lambda \mathrm{nm}\left(\varepsilon \times 10^{-3}\right.$, $\left.\mathrm{M}^{-1} \mathrm{~cm}^{-1}\right)$ in DMSO) $259(1.44), 301 \mathrm{~nm}(0.29)$. F [-IR (KBr pellet. $\mathrm{cm}^{-1}$ ) 3371, 1604, 1526, 1418, 718, 685, 669.

Synthesis of $\mid \mathrm{Er}$ (pentafluorobenzoate) $)_{3}\left(\mathrm{H}_{2} \mathrm{O}\right)_{3} \mid$ (F5-6). According to the similar procedure above. Er(pentafluorobenzoate $)_{3}\left(\mathrm{H}_{2} \mathrm{O}\right)_{3}$ as white solid $(0.422 \mathrm{~g}, 0.494 \mathrm{mmol}, 63 \%)$ was prepared from the reaction of $\mathrm{ErCl}_{3}(0.215 \mathrm{~g} .0 .785$ mol) in methanol $(10 \mathrm{~mL})$ with pentafluorobenzoic acid $(0.500 \mathrm{~g}, 2.357 \mathrm{mmol})$ and $\mathrm{KH}(0.094 \mathrm{~g} .2 .357 \mathrm{mmol})$ in ]HF (100 mL). Anal. (\%) Calc. for $\mathrm{C}_{21} \mathrm{H}_{6} \mathrm{ErF}_{15} \mathrm{O}_{9}$ : C 29.52. $\mathrm{H} 0.71$, Er 19.57; Found: C. 29.41, H 0.72, Er 19.50. UV-vis. $\left(\lambda \operatorname{nm}\left(\varepsilon \times 10^{-3}, \mathrm{M}^{-1} \mathrm{~cm}^{-1}\right)\right.$ in DMSO) $271(4.23), 277(3.51)$ nm. FT-IR (KBr pellet, $\mathrm{cm}^{-1}$ ) 3404, 2991, 2937, 1651, 1617. $1528,1488,1409,1114,1028,996,773,749$.

Synthesis of $\mid \mathrm{Fr}$ (benzoate) ${ }_{3}$ (bipyridine)| (H5-8). To a mixture of benzoic acid $(0.200 \mathrm{~g}, 1.637 \mathrm{mmol})$, bipyridine $(0.255 \mathrm{~g}, 1.637 \mathrm{mmol})$ and $\mathrm{KH}(0.066 \mathrm{~g}, 1.654 \mathrm{mmol}), 100$ $\mathrm{mL}$ of THF was transferred through camnula and the suspension was stirred at room temperature for overnight. A suspension was formed during reaction with gas evolution. After the complete formation of salts, the methanol solution of anhydrous $\mathrm{CrCl}_{3}(0.149 \mathrm{~g}, 0.545 \mathrm{mmol})$ in methanol (10 $\mathrm{ml}$.) was added dropwise to the reaction solution. After 2 days of stirring at room temperature, the resulting solution was filtered and removed solvents to give a white solid. The solid was sequentially washed with methanol, n-hexane and diethylether, and then dried to afford Fr(benzoate)(bipyridine) as a white soild $(0.228 \mathrm{~g}, 0.332 \mathrm{mmol}, 61 \%)$. Anal. (\%) Calc. for $\mathrm{C}_{31} \mathrm{H}_{23} \mathrm{Cr}_{\mathrm{N}} \mathrm{N}_{2} \mathrm{O}_{6}$ : C $54,21, \mathrm{H} 3.38, \mathrm{~N} 4.08$, T.r 24.35; Found: C. 54.25, H 3.42, N 4,01, ए.r 24.33. UV-vis. $\left(\lambda \mathrm{nm}\left(\varepsilon \times 10^{-3}, \mathrm{M}^{-1} \mathrm{~cm}^{-1}\right)\right.$ in DMSO $279(18.45) \mathrm{nm}$. FT-JR (KBr pellet, $\mathrm{cm}^{-1}$ ) 3060, 1640, 1600, 1540, 1410, 1310, $1181,1160,1070,1010,860,770,720,690,670,570,430$.

Synthesis of |Er(pentafluorobenzoate),(bipyridine)| (F5-8). Through the similar procedure for synthesis of H5-8. Er(pentafluorobenzoate) (bipyridine) as a white solid $(0.185$ g, $0.193 \mathrm{mmol}, 61 \%$ ) was prepared from the reaction of $\mathrm{ErCl}_{3}(0.086 \mathrm{~g}, 0.314 \mathrm{mmol})$ in methanol $(10 \mathrm{~mL})$ with pentafluorobenzoic acid $(0.200 \mathrm{~g} .0 .943 \mathrm{mmol}), \mathrm{KH}(0.037$ g, $0.943 \mathrm{mmol})$ and bipyridine $(0.147 \mathrm{~g}, 0.943 \mathrm{mmol})$ in IHF (100 mL). Anal. (\%) Calc. for $\mathrm{C}_{31} \mathrm{H}_{8} \mathrm{ErF}_{15} \mathrm{~N}_{2} \mathrm{O}_{6}: \mathrm{C}$ $38.92, \mathrm{H} 0.84, \mathrm{~N} 2.93, \mathrm{Er} 17.48$; found: $\mathrm{C}, 39.12, \mathrm{H} 0.97, \mathrm{~N}$ 2.75, Er 17.35. UV-vis. $\left(\lambda \mathrm{nm}\left(\varepsilon \times 10^{-3}, \mathrm{M}^{-1} \mathrm{~cm}^{-1}\right)\right.$ in DMSO) 283 (19.94) nm. F"I-IR (KBr pellet. $\left.\mathrm{cm}^{-1}\right) 3100$. $2930,1650,1610,1600,1490,1400,1320,1300,1110$, 1070, 1000, 940, 770, 700,650, 510 .

Photophysical Property Measurement. Photoluminescence spectra were measured by steady-state fluorimeter (Edinburgh FS920) with $450 \mathrm{~W}$ Xe-lamp (295 or $320 \mathrm{~nm}$ ), $\mathrm{He}-\mathrm{Cd}$ laser $(325 \mathrm{~nm})$, and Ar-ion laser (488 nm). The excitation light selected by $300 \mathrm{~mm}$ focal length monochromator was focused on the sample. The fluorescence 
from the sample was collected and refocused to the emission monochromator with $300 \mathrm{~mm}$ focal length. Visible emission spectra were taken with a PMT system (Hamamatsu R955) and near infrared emission spectra were taken with liquid nitrogen cooled Ge-detector (Fdinburgh F.I-].). The excitation laser powers were adjusted to the same value at the sample with neutral density (ND) filters. All spectra were taken at room temperature.

\section{Results and Discussion}

Fr(11])-chelated prototype complexes were carried out through the ligand-exchange reaction using $\mathrm{FrCl}_{\hat{s}}$. At first. the salts of benzoate or pentaflurobenzoate were prepared from the reaction of the organic ligands with $\mathrm{KH}$ in dry THF and then the corresponding salts of the ligands reacted with FrCl: The successful synthesis of $\mathrm{Er}(\mathrm{III})$-chelated prototype complexes was proved by elementary analysis, thermal gravimetric analysis (TGA), FT-IR, absorption and emission spectroscopies. But, the paramagnetic properties of $\mathrm{Sr}$ complexes do not permit NMR characterization of the model complex. FT-IR spectra of [r(III) carboxylate complexes showed two characteristic bands in the region of 1650-1604 and $1418-1395 \mathrm{~cm}^{-1}$ (see Figure 1). ${ }^{1 \cdot 1}$

As seen from Figure 2, at least three $\mathrm{H}_{2} \mathrm{O}$ molecules coordinated in the unsaturated 6-coordinated complexes H5-

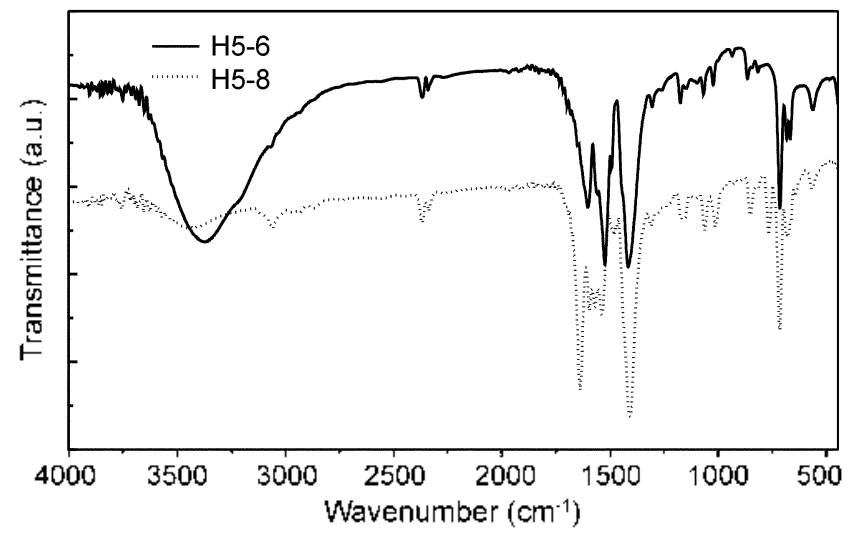

Figure 1. FT-IR spectra of II5-6 and II5-8.

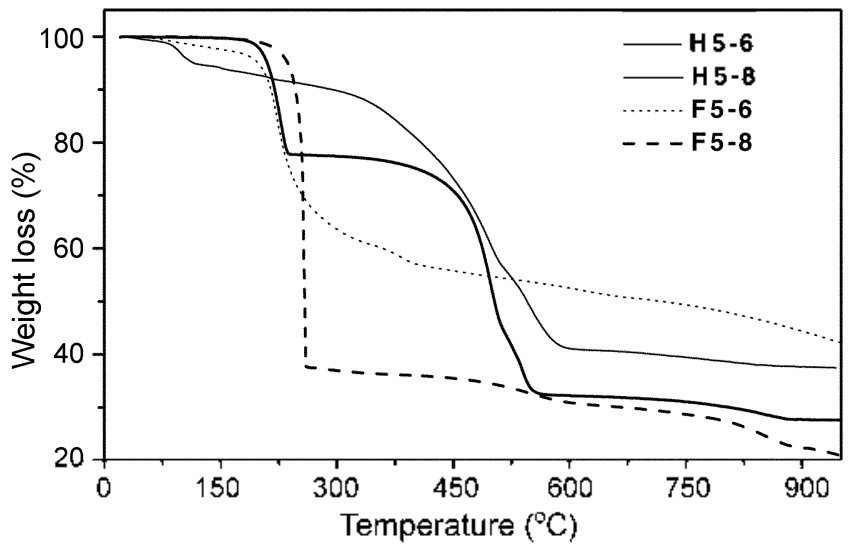

Figure 2. IGA traces of H5-6, H5-8, F5-6 and F5-8.
6 and F5-6, which was confirmed by determining the releasing water amount of $6 \%$ up to $190^{\circ} \mathrm{C}$ in atmospheric condition in a TGA trace. However, the 8-coordinated Fr(III)-chelated complex H5-8 shows two obvious weight loss processes in TGA curve. The first one with the weight loss of $23 \%$ is assigned to the releasing of bipyridine from $170^{\circ} \mathrm{C}$ to $237^{\circ} \mathrm{C}$, and the second one is assigned to the loss of benzoate ligands up to $\mathrm{ca} .550{ }^{\circ} \mathrm{C}$. The 8 -coordinated Er(III)-chelated complex F5-8 also shows one weight loss process in TGA curve, all pentafluorobenzoate and bipyridine liagnds are simultaneously released from $170^{\circ} \mathrm{C}$ to 260 ${ }^{10} \mathrm{C}$. Therefore, 8-coordinated model complexes H5-8 and F5-8 showed no releasing water up to $170^{\circ} \mathrm{C}$, indicating that 8 -coordinated model complexes contain no coordinated $\mathrm{H}_{2} \mathrm{O}$ molecules. Here, for the first time to our knowledge, the first inert, saturated 8-coordinated Fr(111)-chelated complexes of Fr(benzoate) (bipyridine) H5-8 and $\operatorname{\Gamma r}$ (pentafluorobenzoate) (bipyridine) F5-8 were investigated. It is also very important for the formation of inert, saturated erbium complexes to get a higher near IR emission (see below).

The absorption spectra of 8-coordinated prototype complexes H5-8 and F5-8 show a very intense absorption band at $310 \mathrm{~nm}$, which is attributed to $\pi-\pi^{*}$ electronic transition, respectively. The emission spectra of all compexes at the selected photoexcitation wavelength exhibit the emission bands in the near IR region of $1.470-1.630 \mu \mathrm{m}$, which are assigned to the characteristic ${ }^{1} \mathrm{I}_{15: 2} \rightarrow{ }^{4} \mathrm{I}_{152}$ transition of trivalent erbium ions. Figure 3 shows the the emissoin spectra of the 6- and 8-coordinated complexes at the photoexcitation wavelength of 295 or $320 \mathrm{~nm}$. The saturated 8 -coordinated complexes show much stronger PI intensity than that of the unsaturated 6-coordinated complex as shown in Figure 3. The similar result was obtained in a previous report." The excitation spectra of the 8 -coordinated prototype complexes (H5-8 and $\mathbf{F 5 - 8}$ ), monitored at the maximum emission band, show a strong maximum band at $335 \mathrm{~nm}$ in the utraviolet region, whereas the excitation

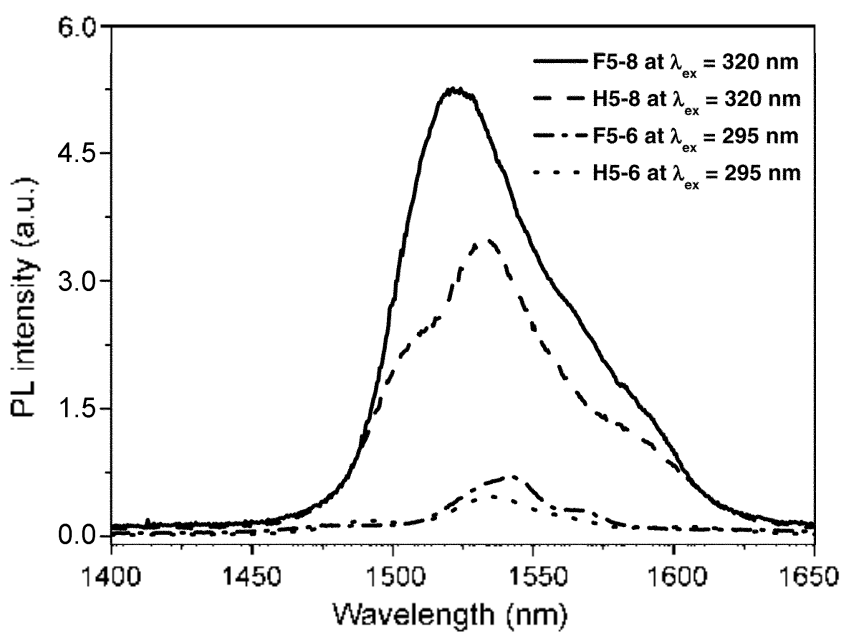

Figure 3. Neas IR emission spectua of the 6- or 8-coordinated model complexes for $\mathbf{F 5 - 8}$ at $\lambda_{\text {cm }}=320 \mathrm{~nm}$. $\mathbf{H 5 - 8}$ at $\lambda_{\text {cmm }}=320 \mathrm{~mm}$. F5-6 at $\lambda_{\mathrm{em}}=295 \mathrm{~nm}$. and $\mathbf{1 5 - 6}$ at $\lambda_{\mathrm{em}}=295 \mathrm{~nm}$ in a solid statc. Here. the $\hat{\lambda}_{\mathrm{ex}}$ means the excitation wavelength. 


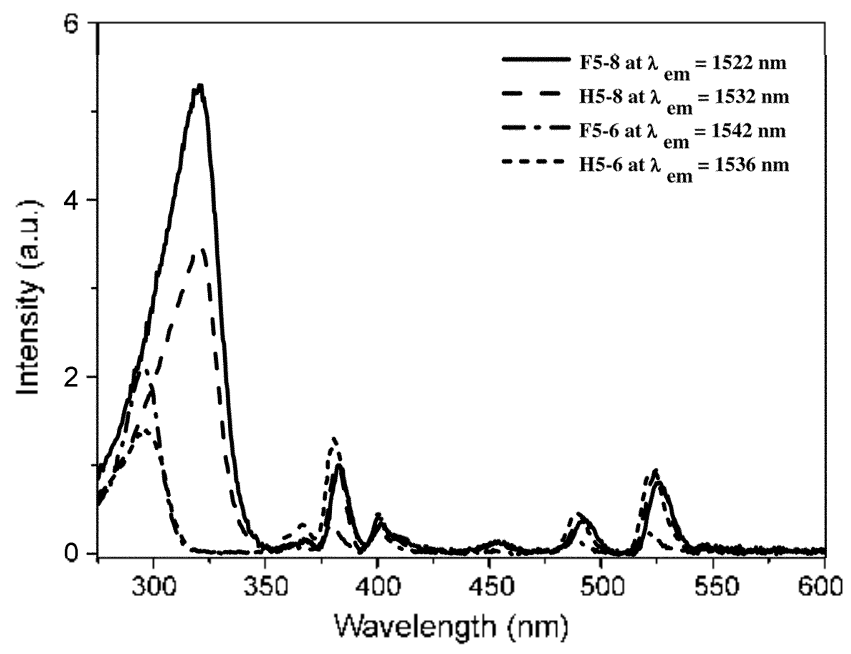

Figure 4. Lxcitation spectra of $\mathbf{1 5 - 8}$ at $\lambda_{\mathrm{em}}=1522 \mathrm{~nm}$. H5-8 at $\lambda_{\mathrm{em}}$ $=1532 \mathrm{~nm}$. F5-6 at $\lambda_{\mathrm{em}}=1542 \mathrm{~nm}$. and F5-6 at $\lambda_{\mathrm{em}}=1536 \mathrm{~nm}$ in solid state. Hete. the $\lambda_{\text {en }}$ means the emission wavelength.

spectra of the 6-coordinated complexes of H5-6 and F5-6 exhibit the bands at $295 \mathrm{~nm}$ (see Figure 4). Also, the several additional excitation bands were exhibited in the region of $350-550 \mathrm{~nm}$. These excited bands are consistent with the absorption bands of erbium ions. ${ }^{1.8}$ Thus, these excited bands correspond to the absorption bands of the inherent Fr(III) ion. With these results, it should be noted to spectrally visualize that the energy transfer between the excited organic ligand and the ground erbium ion takes place (Figure $3 \& 4$ ).

Figure 5 shows the near IR emission bands of the 6 coordinated and 8-coordinated prototype complexes, corresponding to the characteristic $\left.\left.{ }^{1 /}\right]_{132} \rightarrow{ }^{1}\right]_{15: 2}$ transition of trivalent erbium ions taking place at $1.53 \mu \mathrm{m}$, upon a photoexcitation wavelength of 325 or $488 \mathrm{~nm}$. Here, upon the photoexcitation wavelength of $325 \mathrm{~nm}$, the energy transfer process between the excited ligand and Er ion takes

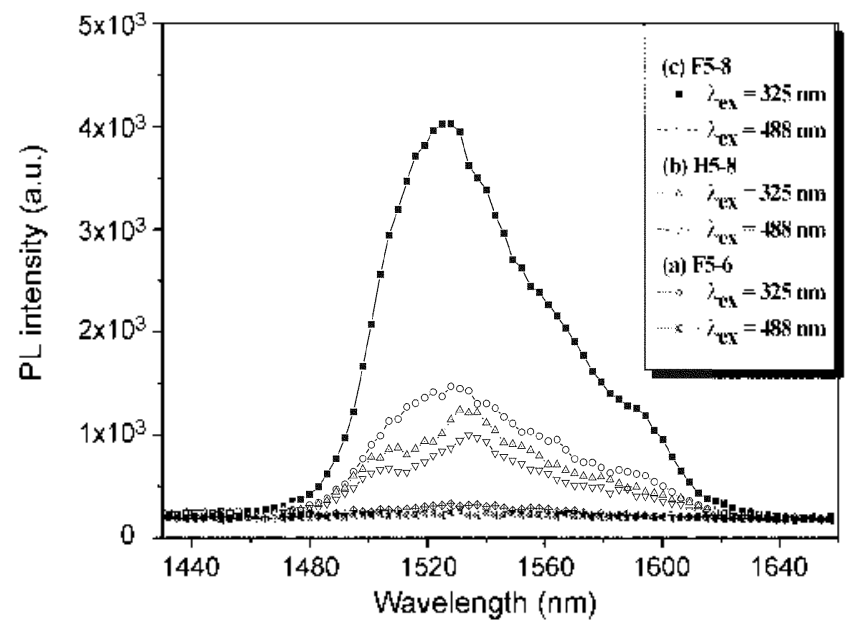

Figure 5. Vear IR emission spectra of the 6- or 8-coordinated model complexes for (a) $\mathbf{1 5 - 6}$. (b) H5-8. and (c) $\mathbf{5 - 5}$ in a solid stat:. place possibly to generate the emission of the central lanthanide ion ( $\Gamma$ r jon), while, upon the photoexcitation wavelength of $488 \mathrm{~nm}$, the emission of the inherent lanthanide ion ( $\mathrm{\Gamma r}$ ion) was obtained through the direct excitation of Fr ion without the energy transfer process between the excited ligand and Fr ion. The saturated 8coordinated complexes show much stronger $\mathrm{Pl}$. intensity than that of the unsaturated 6-coordinated complex as shown in Figure 5, in which the highly coordinated lanthanidechelated complexes (at least 8 to 9 coordination) have the higher PI, efficiency than the unsaturated lanthanidechelated complexes ( 6 coordination). ${ }^{\text {" In }}$ the latter complex. at least three $\mathrm{H}_{2} \mathrm{O}$ molecules coordinated in the unsaturated 6-coordinated complexes seriously quenched the near IR emission by the hamonic vibration relaxation decay of $\mathrm{O}-\mathrm{H}$ bonds in these $\mathrm{H}_{2} \mathrm{O}$ molecules. It reduces greatly the intensity of the near IR emission. Also, the saturated 8coordinated complexes, obtained with a photoexcitation wavelength at $325 \mathrm{~nm}$, exhibit much higher PL intensity than that obtained from the direct photoexcitation of Er ions with $488 \mathrm{~nm}$ by 80 times.

$$
W_{M / n}=W_{1} \exp \left[-\frac{\Delta E}{h \omega}\left(\ln \frac{\Delta E}{h \omega} \frac{1}{g(n+1)}-1\right)\right]
$$

Furthermore, we have investigated the influence of $\mathrm{C}-\mathrm{F}$ bonds on near IR emission by comparing the PI, intensity of a 8-coordinated model complex with C-F bonds (F5-8) with that of a 8 -coordinated model complex with $\mathrm{C}$-H bonds (H58). The PI intensity at the near IR emission is proportional to the density of excited ions in the upper energy level (" $]_{13: 2}$ ). The higher the excited ion density increases, the stronger the PL, intensity at the near IR emission obtains. Therefore, it is necessary to enhance the excited ion density in upper energy level for getting the stronger near IR emission intensity. The excited ion density in the upper energy level ( ${ }_{1,3}$ ) strongly depends on the multi-phonon relaxation process. The multi-phonon relaxation process is one of the nonradiative transitions, where the photon energy is absorbed by the harmonic vibration motion to convert to the themal energy, instead of light emission. The probability for the multi-phonon relaxation ( $W_{\mathrm{Y} \mid P R}$ ) is related to the energy difference $(\Delta \mathrm{E})$ between the upper level and the lower level, as given in eq. (1), where $\mathrm{g}$ and $\mathrm{n}$ are the bond constant of electron-lattice and Bose-Einstein distribution function, respectively. ${ }^{15}$ If the energy difference $(\Delta \mathrm{E})$ decreases, $W_{Y, 1 / K}$ exponentially increases. When the harmonic vibration energy (hw) of the material matrix decreases. $W_{\text {NIPR }}$ also decreases exponentially.

In the case of the $\mathrm{Er}^{3+}$ ions, the energy difference $(\Delta \mathrm{E})$ between the upper level $\left({ }^{4} I_{1} s_{2}\right)$ and the nearest lower level $\left({ }^{4}\right\rfloor_{15 i 2}$ ) corresponds to the wavenumber of $7,000 \mathrm{~cm}^{-1}$ (or the wavelength of $1.53 \mu \mathrm{m})$. Er(II])-cored model complex with $\mathrm{C}-\mathrm{F}$ bond has a very low $\mathrm{W}_{\mathrm{MPR}}$, because the harmonic vibration energy (ha) of the C-F bonds is much lower than this energy difference. However, in $\mathrm{Er}(\mathrm{Il}[\mathrm{L}$-cored benzoate complex, the harmonic vibration energy of $\mathrm{C}-\mathrm{H}$ overtone bands is similar to the energy difference $(\Delta \mathrm{E})$ of $7,000 \mathrm{~cm}^{-1}$. 
Therefore. it causes the increase of $\mathrm{W}_{\mathrm{M} F \mathrm{r}}$, thus decreases the near IR emission intensity. In line with this reason. Figure 5 shows that F5-8 has much stronger PL intensity than that of H5-8 by four times. In this paper. our main material efforts have been focused on developing the new synthetic methodolgy of the inert, saturated Er conplexes and investigating important key parameters for near IR enission enhancement. The new synthetic methodolgy opens the development of a series of novel lanthanide-cored nanophotonics systems based on porphyrins. and naphthalenes with highly efficient light-harvesting effect. ${ }^{1.10}$

\section{Conclusion}

We have synthesized the $\operatorname{Er}(\mathrm{III})$-chelated prototype complexes based on benzoate derivatives through a new synthetic strategy developed in our laboratory and investigated the important key parameters for near IR emission enhancement. The saturated 8-coordinated Er(III) complexes exhibit stronger near-IR emission bands than those of the unsaturated 6-coordinated Er(III) coniplexes. obtained from the direct photoexcitation of $\mathrm{Er}$ ions with $488 \mathrm{~nm}$. At least three $\mathrm{H}_{2} \mathrm{O}$ molecules coordinated in the unsaturated 6coordinated complexes seriously quenched the near IR emission by the harmonic vibration relaxation decay of $\mathrm{O}-\mathrm{H}$ bonds in these $\mathrm{H}_{3} \mathrm{O}$ molecules. It reduces greatly the intensity of the near IR enission. which takes place from the first excited state $\left({ }^{4} \mathrm{I}_{13: 2}\right)$ to the ground state $\left({ }^{4} \mathrm{I}_{15: 2}\right)$ at 1.53 $\mu \mathrm{m}$. Also. the stronger emission band of the Er(III) complex was obtained by the indirect photoexcitation of ligands rather than by the direct photoexcitation of the Er(III) ions. It could be explained by the energy transfer between PL emission band of ligands and absorption band of erbium ion. The saturated $\operatorname{Er}(\mathrm{III})$-chelated complex with C-F bonds shows much stronger near IR emission than the saturated $\mathrm{Er}$ (III)-chelated complex with $\mathrm{C}$-H bonds. It is attributed to the influence of $\mathrm{C} \cdot \mathrm{F}$ bonds on near IR emission intensity.
Acknowledgements. The authors acknowledge that this research was funancially supported from both the National Creative Research Initiatives Program of Ministry of Science and Teclunology of Korea and Hannam University (Kyobi 2003).

\section{References}

1. Kim. H. K.: Roh. S. G.: Hong. K.-S.: Ka. J.-W.: Baek. N. S.: Oh. J. B.: Nah. M. K.: Ma. S. M.: Cha. Y. H.: Ko. T. Hacromol Res. 2003. $H(3), 133$ and see references cited therein.

2. Steemers. F. J.: Verboom. W.: Reinhoudt. D. N.; van der Tol, E. B.; Verhoeven, J. W. J. Am. Chent. Soc, 1995, 117, 94018.

3. Oude Wolbers. M. P.: vant Veggel. F. C. T. M.: Peters. F. G. A.: van Beelet1. E. S. E.: Hotstraat. J. W.: Guerts. F. A. J.: Reinhoudt. D. N. Chem. Em. J. 1998. 4. 772.

4. Slooff, L. H.: Polman, A.: Oude Wolbers, M. P.; wan Veggel, F. C. J. M.: Reinhoudt. D. N.: Hofstraat, J. W. J. Appl. Phts 1998, 83. 497.

5. Klink. S. I.: Hebbitnk. G. A.: Grave. L.: yan Veggel. F. C. J. M.: Reinhoudt. D. N.: Slootf. L. H. J. Appl. Plys. 1999. 86. 1181.

6. Pitois. C.: Vestberg. R.: Rodlert. M.: Malmiström. E.: Hult. A.: Lindgren. M. Opt A fater, 2002, 21, 499.

7. Destri. S:; Porzio. W.; Meinardi. F:: Tubino. R; Salemo. G. Macrontolecules 2003. 36,273

8. Roh. S. G.: Baek. N. S.: Hong. K.-S.: Kim. H. K. Bull. Konam Chem. Soc. 2004. 25. 343.

9. Oh. J. B.: Paik. K. L.: Ka. J.-W.: Roh. S.-G.: Nah. M.-K.: Kim. H K. Mater Sci \& Eng C 2004. 24. 257.

10. (a) Baek. N. S.: Nah. M.-K.: Kim, Y. H.: Roh. S. G.: Kim, H. K. Bull. Korean Chem. Soc. 2004, 25, 443. (b) Kim, H. K.: Oh, J. B.; Baek. N. S.: Ka. J.W.: Roh. S. G.: Kim. Y. H.: Nah. M. K.: Hong. K.-S.: Song. B. I.: Zhou. G. J. Yonlhear Optical Phusics \& Materials. accepted. 2004.

11. Kawa, M.: Frechet. M. J. Chent Mater: 1998. 10. 286

12. Paik, K. L: Kim, H. K. Mol Cnst \& Liq. Chst 2001. $370,185$.

13. Kim. H. K.: Roh, S. G.: Ka, J. W.: Kim. Y. H.: Nah, M. K. Oh. J B.: Baek. N. S. Korean Patent and International PCT Patent filed (Janluary 31. 2004: PCT KR20044:000181).

14. Rardin. R. L.: Tolman: W. B.: Lippard. S. J. New d. Chem 1991. 15. 417 .

15. Desurvire, E. Erbint-doped Fiber Amplifiers: Principles and Appications; John Wiley \& Sons: New York, 1994: Chapter 4. 